\title{
Associations between a priori-defined dietary patterns and longitudinal changes in bone mineral density in adolescents
}

\author{
Teresa Monjardino ${ }^{1,2, *}$, Raquel Lucas ${ }^{1,2}$, Elisabete Ramos ${ }^{1,2}$ and Henrique Barros ${ }^{1,2}$ \\ 'Department of Clinical Epidemiology, Predictive Medicine and Public Health, University of Porto Medical \\ School, Al Prof Hernâni Monteiro, 4200-319 Porto, Portugal: ${ }^{2}$ Institute of Public Health of the University of Porto, \\ Porto, Portugal
}

Submitted 24 March 2012: Final revision received 18 September 2012: Accepted 21 September 2012: First published online 13 November 2012

\begin{abstract}
Objective: To quantify short- and long-term associations between dietary patterns defined a priori and bone mineral density (BMD) during adolescence.

Design: Dietary patterns were defined at 13 years old using a Mediterranean diet (MD) quality index, the Dietary Approaches to Stop Hypertension (DASH) diet index and the Oslo Health Study (OHS) dietary index. Linear regression coefficients were used to estimate associations between dietary patterns and forearm BMD at 13 and 17 years, measured by dual-energy X-ray absorptiometry. Setting: Public and private schools of Porto, Portugal.

Subjects: The EPITeen cohort comprising 1180 adolescents born in 1990, recruited at schools during the 2003/2004 school year and re-evaluated in $2007 / 2008$.

Results: In girls, at 13 years, mean BMD $\left(\mathrm{g} / \mathrm{cm}^{2}\right)$ in the first and third tertiles was 0.369 and 0.368 for the MD pattern, 0.368 and 0.369 for the DASH diet, and 0.370 and 0.363 for the OHS index. In boys, mean BMD $\left(\mathrm{g} / \mathrm{cm}^{2}\right)$ in the first and third tertiles was 0.338 and 0.347 for the MD pattern, 0.342 and 0.346 for the DASH diet, and 0.344 and 0.342 for the OHS index. None of these differences were significant. Mean BMD at 17 years and prospective variation were also not significantly different between tertiles of adherence to each score. However, a trend of increased BMD at 13 years with greater adherence to the MD pattern was observed in boys (adjusted coefficient $=0 \cdot 248 ; 95 \%$ CI 0.052, 0.444).

Conclusions: The selected dietary patterns may not capture truly important dietary differences in determining BMD or diet may not be, beyond nutrient adequacy, a limiting determinant of BMD.
\end{abstract}

Keywords
Dietary patterns
Bone density
Cohort study
Adolescence

Dietary patterns

Cohort study

Adolescence
Osteoporotic fractures are a major cause of morbidity and mortality whose burden is expected to increase in the future $^{(1,2)}$. Research has been focused on understanding the mechanisms of age-related bone loss, but growing evidence emphasizes the influence of early-life factors in the attainment of adult bone mass ${ }^{(3,4)}$. It has been suggested that high peak bone mass protects against fragility fractures later in life ${ }^{(3-5)}$. Diet is known to modulate achievement of the full genetic potential for skeletal mass during adolescence ${ }^{(4,6)}$ but research has typically examined the effects on bone mineral density (BMD) of single nutrients or foods (e.g. Ca, P, vitamin D, vitamin K and protein) or food groups such as dairy products, fruit and vegetables ${ }^{(7-9)}$. However, meals consist of a variety of foods with complex combinations of nutrients that are likely to be interactive and many foods and nutrients are highly correlated, making it difficult to separate their effects. Effects of single nutrients may be too subtle to detect with the available measurement instruments, but combined effects as expressed in a dietary pattern may be sufficiently large.

Dietary patterns analysis is a more comprehensive approach to dietary exposure assessment and to examine its relationship with bone health ${ }^{(10,11)}$. Dietary choices in epidemiological studies may be assessed through compliance with dietary patterns defined a priori based on presumed health effects ${ }^{(12)}$. Indices to measure adherence to the Mediterranean diet (MD) have been used to explore the risk of obesity, CVD and cancer ${ }^{(13)}$. Although some components of this pattern have been associated with bone quality in children $^{(14)}$, the overall effect of adherence to this type of diet on bone accrual remains unknown.

The Dietary Approaches to Stop Hypertension (DASH) diet is a Ca-rich diet that emphasizes fruits, vegetables and low-fat dairy products ${ }^{(15-17)}$. Although originally designed for blood pressure reduction, several aspects of this pattern may benefit bone such as the rich $\mathrm{Ca}, \mathrm{K}$ and Mg contents ${ }^{(18)}$. 
MD and DASH do not specifically assess foods with a possible negative effect on bone quality. Such information is of major interest and can be obtained with the Oslo Health Study (OHS) dietary index built on prevailing knowledge about foods that potentially influence bone health. The OHS is computed as the ratio between soft drinks and fruit and vegetables consumption and has been directly associated with metabolic syndrome and inversely associated with $\mathrm{BMD}^{(19,20)}$.

In addition to being a critical period in the acquisition of bone mass ${ }^{(5)}$, adolescence is also a period of social and psychological influences on food choices ${ }^{(21)}$.

To the best of our knowledge, there are no studies examining the association between a priori-defined dietary patterns and BMD in adolescents. By using prospective data from a population-based cohort, our objective was to quantify the associations between forearm BMD in early and late adolescence and adherence to a priori-defined dietary patterns in early adolescence, using the Mediterranean Diet Quality Index for children and adolescents (KIDMED), the DASH diet index and the OHS dietary index.

\section{Experimental methods}

The EPITeen cohort was assembled during the 2003/2004 school year, when all public and private schools in Porto that provided teaching to adolescents born in 1990 were approached. Nineteen private and all public schools $(79 \cdot 2 \% v \cdot 100 \%, P=0 \cdot 04)$ agreed to participate in the study. Executive boards were asked to provide contact information for each student's family. Parents received written explanation of the purpose and the study and meetings were arranged in each school in order to explain research aims and procedures. We identified 2787 eligible adolescent students, of whom 2160 (1044 boys) agreed to participate and provided information for at least part of the protocol $(77.5 \%$ participation at the individual level). Similar participation proportions were obtained in public and private schools. The follow-up evaluation of the cohort took place in the 2007/2008 school year and 1716 participants were successfully re-evaluated $(79 \cdot 4 \%)$. Sampling procedures and detailed methods have been described elsewhere ${ }^{(22)}$. The study was approved by the Ethics Committee of the University Hospital of São João, Porto and policies and procedures were developed to guarantee data confidentiality and protection. Written informed consent was obtained from both parents and adolescents.

\section{Physical examination}

In both evaluations, forearm BMD $\left(\mathrm{g} / \mathrm{cm}^{2}\right)$ was measured at the distal radius of the non-dominant forearm by dualenergy X-ray absorptiometry (DXA) using a Lunar ${ }^{\circledR}$ PIXI device (GE Medical Systems, Madison, WI, USA). In cases of reported previous fracture of the non-dominant arm, the dominant arm was assessed. Anthropometry was obtained while the student stood barefoot in light indoor clothing. Weight was measured using a digital scale to the nearest tenth of a kilogram (Tanita Corporation, Tokyo, Japan) and height was measured in centimetres, to the nearest tenth, using a portable stadiometer (Seca Deutschland, Hamburg, Germany); BMI was calculated.

\section{Home questionnaires variables}

At 13 years of age, dietary intake was evaluated using an FFQ designed according to Willett et $a l^{(23)}$ and adapted for the Portuguese population and previously validated in Porto adults ${ }^{(24,25)}$. The FFQ was adapted for adolescents through the addition of foods more frequently eaten by this age group ${ }^{(26,27)}$. The FFQ comprised ninety-one food items and an open-ended section to add items not listed in the questionnaire but eaten at least once per week. Participants were asked to report, over the prior 12-month period, the frequency of consumption of each food in one of nine categories, ranging from 'never or less than once per month' to 'six or more times per day'. The FFQ did not include specific questions on portion size and the frequency reported was multiplied by a standard portion size to estimate the average daily intake. Seasonal variation of food consumption was considered by multiplying the frequency and portion of seasonal items by a seasonal factor of $0 \cdot 25$ (i.e. equivalent to consumption during a 3-month period). Food consumption was converted into energy and nutrient intakes with the software program Food Processor Plus (1997; ESHA Research, Salem, OR, USA) based on values from the US Department of Agriculture and which was updated with values for typical Portuguese foods ${ }^{(24)}$.

Information on regular practice of physical activity at 13 years was quantified through the frequency spent in sport activities of at least twenty consecutive minutes beyond compulsory school activities. Adolescents were classified according to parental educational level measured as the number of successfully completed years of formal schooling of the parent with higher education. Since examination at school limited conditions to evaluate adolescents' pubertal development according to Tanner stages $^{(28)}$, age at menarche referred at 13 years of age was recorded as a pubertal development indicator for girls.

\section{Data analysis}

The degree of adherence to the MD pattern was measured using the Mediterranean Diet Quality Index for children and adolescents (KIDMED) ${ }^{(29)}$; adherence to the DASH diet was evaluated through a DASH diet index score proposed by Fung and colleagues ${ }^{(30)}$; and the total score of the OHS dietary index ${ }^{(20)}$ was calculated.

The original KIDMED includes sixteen components. Items denoting a negative connotation with respect to the MD are assigned a value of -1 , and those with a positive 
aspect are assigned +1 . This index assesses the daily consumption of at least one serving of fruit and vegetables, rating higher when consumption is greater. Weekly consumption of at least two to three servings of nuts and fish and of more than one serving of pulses is evaluated. It also assesses the consumption of pasta or rice at least five times weekly; grains or cereals, daily for breakfast; dairy products, three servings daily; and olive oil for culinary use. Items denoting a negative connotation with respect to the MD include frequent intake of sweets, candy, commercially baked goods, pastries, fast foods, and non-consumption of breakfast ${ }^{(29)}$. The higher the KIDMED score, the more Mediterranean and favourable is the dietary pattern. We eliminated the items regarding breakfast since our dietary evaluation was based on an FFQ. Excellent agreement $(\kappa=0 \cdot 86)$ between the original KIDMED and the adapted version was found in a different sample of 117 adolescents aged 13 years.

We used a DASH score awarding points for high intake of fruit, vegetables, nuts, legumes, dairy products and whole grains according to quintile rankings (i.e. participants in the lowest quintile were assigned 1 point and those in the highest quintile were assigned 5 points). Regarding the intake of $\mathrm{Na}$, red and processed meat, and sweetened beverages, participants in lower quintiles of intake scored higher ${ }^{(30)}$. Higher scores reflect higher compliance with DASH characteristics.

The OHS dietary index was constructed based upon the literature about foods that potentially influence $\mathrm{Ca}$ balance or acid load, and consequently bone health. It is a ratio between the intake of soft drinks (negative for bone health owing to its role in acid load increase and promotion of urinary loss of $\mathrm{Ca}$ ) and the intake of fruit/ berries, fruit juice and cooked and raw vegetables (positive for bone owing its role in promotion of $\mathrm{Ca}$ absorption and reduction of $\mathrm{Ca}$ loss $)^{(20)}$. Thus, it is expected that higher scores on this index, reflecting unhealthier patterns, are negatively associated with BMD. Our index was based on the sum of intake frequency categories of three items (range 3-27): 'colas', 'ice tea soft drinks' and 'other soft drinks', divided by the sum of intake frequency categories of three items (range 3-27): 'vegetables' (fresh or cooked), 'vegetable soup' and 'fruit' (fresh, canned or juice). This adaptation was due to differences in the FFQ used.

Categorical and continuous variables were summarized as percentages and as means and standard deviations, respectively, by tertile of the final KIDMED, DASH diet index and OHS dietary index scores, separately for boys and girls. Differences in proportions were tested with the $\chi^{2}$ test. One-way ANOVA and Kruskal-Wallis one-way ANOVA were used to compare continuous variables between independent samples.

Using adherence to the MD dietary pattern, to the DASH diet and to the OHS dietary index as the main exposures and forearm BMD as the outcome, associations were estimated cross-sectionally (KIDMED score $_{13} v$. $\mathrm{BMD}_{13}$, DASH diet $\operatorname{score}_{13} v \cdot \mathrm{BMD}_{13}$ and OHS dietary index score $_{13} v . \mathrm{BMD}_{13}$ ) and prospectively (KIDMED score $_{13} v$. $\triangle \mathrm{BMD}$, DASH diet score ${ }_{13} v . \Delta \mathrm{BMD}$, OHS dietary index score ${ }_{13} v$. $\Delta$ BMD, KIDMED score 13 v. $\mathrm{BMD}_{17}$, DASH diet score ${ }_{13} v \cdot \mathrm{BMD}_{17}$ and OHS dietary index score ${ }_{13} v$. $\mathrm{BMD}_{17}$ ), using linear regression coefficients and 95\% confidence intervals. BMD was used in $\mathrm{mg} / \mathrm{cm}^{2}$ to improve readability. In addition to crude estimates, linear regression coefficients were also adjusted for height and weight at 13 years and at 17 years old, total energy intake at 13 years old, parental educational level, regular practice of physical activity and mean $\mathrm{Ca}$ intake. In girls, coefficients were further adjusted to menarche age to address confounding by pubertal status.

\section{Sample description}

From a total of 2160 adolescents who participated in the first evaluation, 1264 (591 boys) were included in the cross-sectional analysis (complete information on forearm BMD, anthropometric variables, parental educational level, age at menarche and FFQ). Of those 1264 adolescents, $1075(85.0 \%)$ participated in the follow-up evaluation. The final sample in the longitudinal analyses included 1023 adolescents ( 474 boys) after exclusion of participants with missing information in the follow-up evaluation (Fig. 1).

When participants with and without missing information for any of the above-mentioned variables in the baseline evaluation were compared (896 v. 1264 participants, respectively), we found no differences in female proportion, in BMD and in BMI means at 13 years. However, adolescents with missing information had lower median (25th percentile, 75 th percentile $\left(\mathrm{P}_{25}, \mathrm{P}_{75}\right)$ ) parental educational level $(9(6,12)$ years $v .11(7,16)$ years, respectively; $P<0 \cdot 001)$.

No significant differences in female proportion, in BMD and in BMI means at 13 years were found between participants with complete information in both baseline and follow-up evaluations and participants with missing information (1023 with complete information $v .896$ with missing information in the baseline evaluation +189 lost to followup + fifty-two with missing information in the follow-up evaluation). However, adolescents with complete information had higher median $\left(\mathrm{P}_{25}, \mathrm{P}_{75}\right)$ parental educational level $(12(8,16)$ years $v .9(6,12)$ years, $P<0 \cdot 001)$.

\section{Results}

Mean forearm BMD increased from $0.360($ SD 0.057$) \mathrm{g} / \mathrm{cm}^{2}$ at 13 years to $0.435(\mathrm{sD} 0.052) \mathrm{g} / \mathrm{cm}^{2}$ at 17 years in girls and from $0.342(\mathrm{sD} 0.050)$ to $0.452(\mathrm{sD} 0.075) \mathrm{g} / \mathrm{cm}^{2}$ in boys.

There were no statistically significant differences in mean KIDMED and DASH diet scores by gender (KIDMED score: $5 \cdot 2$ (SD 2.0) for boys, $5 \cdot 1$ (SD 2 1 ) for girls, 


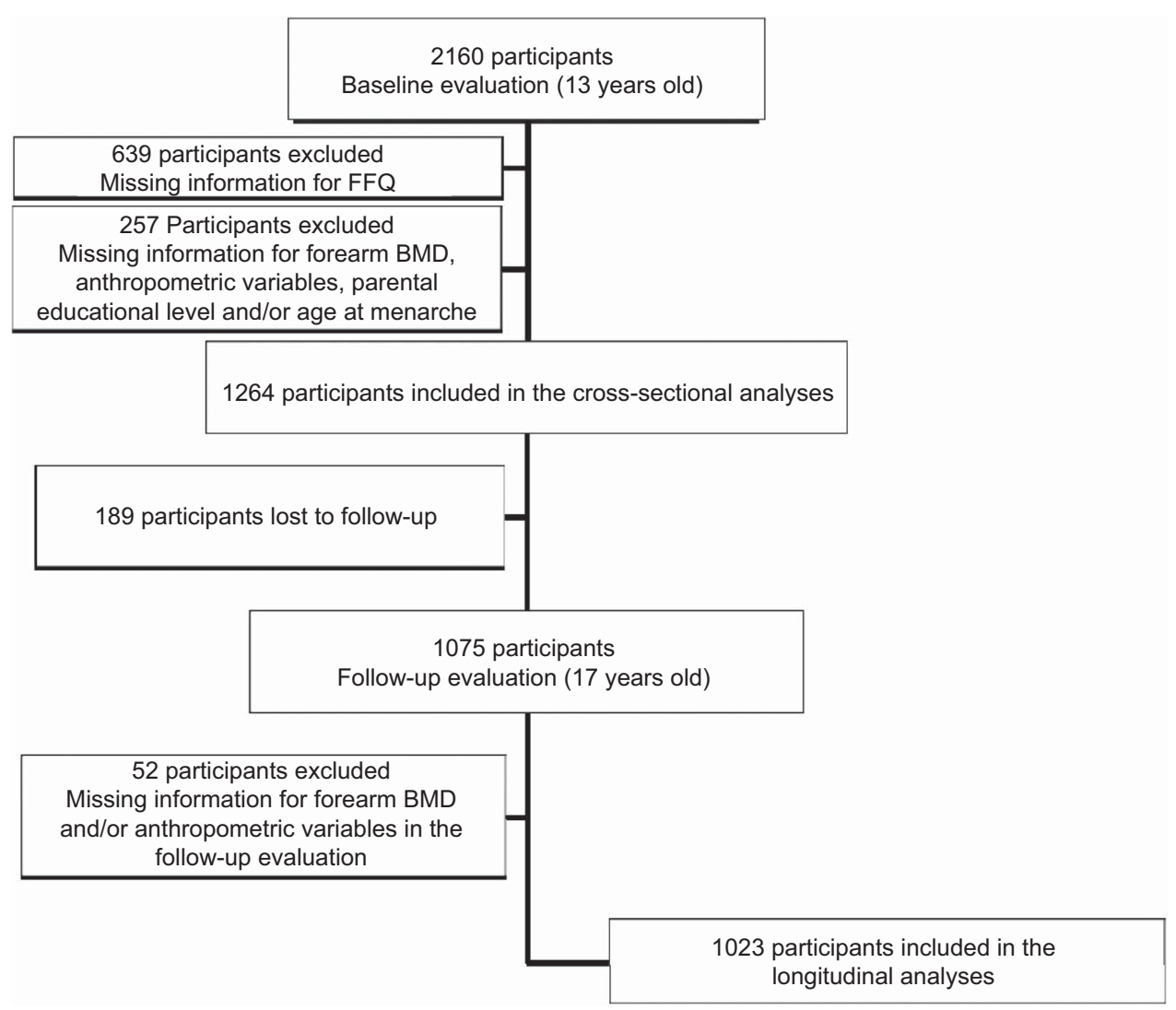

Fig. 1 Flowchart of the included and excluded participants in the cross-sectional and longitudinal analyses (BMD, bone mineral density)

$P=0 \cdot 410$; DASH diet score: $23 \cdot 7$ (sD $4 \cdot 5$ ) for boys, $23 \cdot 7$ (sD 4.5 ) for girls, $P=0 \cdot 908$ ). The mean OHS dietary index score was significantly higher in boys than in girls $(1 \cdot 1$ (SD 0.6) v. $1 \cdot 0(\mathrm{SD} 0 \cdot 5)$, respectively, $P=0 \cdot 005)$.

Average values of anthropometric, sociodemographic and behavioural characteristics in the baseline evaluation, stratified according to tertile of each a priori dietary pattern, are presented in Table 1 for girls and boys. Proportional distributions are presented for categorical variables.

We observed that adolescent girls and boys with healthier dietary patterns, i.e. whose dietary patterns were more similar to the MD pattern, to the DASH diet and who had lower scores in the OHS dietary index, were significantly more likely to have parents with higher education (all $P<0 \cdot 001)$.

For both sexes, adolescents with higher adherence to the MD pattern, with higher adherence to the DASH diet and with lower OHS dietary index scores tended to practise physical activity more frequently. Significantly higher frequency of physical activity was observed in girls when adherence to the MD pattern $(P=0 \cdot 008)$ and the DASH diet $(P=0 \cdot 032)$ was higher and in boys when OHS dietary index score was lower $(P=0 \cdot 038)$.

Although adolescents in the highest tertile of adherence to the MD pattern, as compared with those in the first and second tertiles of adherence, had significantly higher energy intake $(P<0 \cdot 001)$, these adolescents had no statistically significant differences in BMI in the baseline evaluation. We also found no meaningful differences between tertiles of adherence to the MD pattern with regard to mean BMI at 17 years in the follow-up evaluation (data not shown).

As in the baseline evaluation (Table 1), we also found no significant differences in mean follow-up BMI by tertile of adherence to the DASH diet and of OHS dietary index score among males and by tertile of OHS dietary index score among females (data not shown). Although significant differences in girls' mean follow-up BMI by tertile of adherence to the DASH diet have been observed (first tertile: $21 \cdot 7$ (SD 3.4); second tertile: $22 \cdot 6$ (SD 3.5); third tertile: 21.9 (SD 3.2); $P=0.027$ ), a dose-response relationship was not present.

Table 2 shows the average baseline and follow-up BMD for each of the a priori dietary patterns in tertiles. We observed no statistically significant differences in mean BMD, measured in the baseline and in the followup evaluations, or in BMD change by tertile of adherence to the a priori-defined dietary patterns.

Table 3 summarizes the cross-sectional and prospective associations between adherence to the MD pattern, to the DASH diet and to the OHS dietary index and BMD in girls and boys, by presenting crude and adjusted linear 
Table 1 Description of average BMI, regular physical activity, parental educational level and total energy intake at 13 years old (baseline evaluation, 2003/2004) according to tertile of adherence to the Mediterranean diet (MD) pattern, to the Dietary Approaches to Stop Hypertension (DASH) diet and to the Oslo Health Study (OHS) dietary index among Portuguese girls and boys, EPITeen cohort

\begin{tabular}{|c|c|c|c|c|c|c|c|c|c|c|c|c|c|c|c|c|c|c|c|c|c|}
\hline & \multicolumn{21}{|c|}{ Girls (13 years old) } \\
\hline & \multicolumn{6}{|c|}{ MD pattern } & \multirow[b]{3}{*}{$P$} & \multicolumn{6}{|c|}{ DASH diet } & \multirow[b]{3}{*}{$P$} & \multicolumn{6}{|c|}{ OHS dietary index } & \multirow[b]{3}{*}{$P$} \\
\hline & \multicolumn{2}{|c|}{$\begin{array}{l}\text { 1st tertile } \\
\text { (n 247) }\end{array}$} & \multicolumn{2}{|c|}{$\begin{array}{l}\text { 2nd tertile } \\
\text { (n 232) }\end{array}$} & \multicolumn{2}{|c|}{$\begin{array}{l}\text { 3rd tertile } \\
(n 194)\end{array}$} & & \multicolumn{2}{|c|}{$\begin{array}{l}\text { 1st tertile } \\
\text { (n 272) }\end{array}$} & \multicolumn{2}{|c|}{$\begin{array}{l}\text { 2nd tertile } \\
\text { (n 204) }\end{array}$} & \multicolumn{2}{|c|}{$\begin{array}{l}\text { 3rd tertile } \\
(n \text { 197) }\end{array}$} & & \multicolumn{2}{|c|}{$\begin{array}{l}\text { 3rd tertile } \\
(n \text { 226) }\end{array}$} & \multicolumn{2}{|c|}{$\begin{array}{l}\text { 2nd tertile } \\
(n \text { 224) }\end{array}$} & $\begin{array}{c}\text { 1st te } \\
(n 2 \text { ? }\end{array}$ & $\begin{array}{l}\text { ertile } \\
223)\end{array}$ & \\
\hline & $\begin{array}{l}\text { Mean } \\
\text { or } n\end{array}$ & SD or $\%$ & $\begin{array}{l}\text { Mean } \\
\text { or } n\end{array}$ & SD or $\%$ & $\begin{array}{l}\text { Mean } \\
\text { or } n\end{array}$ & SD or $\%$ & & $\begin{array}{l}\text { Mean } \\
\text { or } n\end{array}$ & SD or $\%$ & $\begin{array}{l}\text { Mean } \\
\text { or } n\end{array}$ & SD or \% & $\begin{array}{l}\text { Mean } \\
\text { or } n\end{array}$ & SD or $\%$ & & $\begin{array}{l}\text { Mean } \\
\text { or } n\end{array}$ & SD or $\%$ & $\begin{array}{l}\text { Mean } \\
\text { or } n\end{array}$ & SD or $\%$ & $\begin{array}{l}\text { Mean } \\
\text { or } n\end{array}$ & SD or \% & \\
\hline Median score $\left(P_{25}, P_{75}\right)$ & $3 \cdot 0$ & $2 \cdot 0,4 \cdot 0$ & $5 \cdot 0$ & $5 \cdot 0,6 \cdot 0$ & $7 \cdot 0$ & $7 \cdot 0,8 \cdot 0$ & & $20 \cdot 0$ & $\begin{array}{l}18 \cdot 0 \\
21 \cdot 0\end{array}$ & $24 \cdot 0$ & $\begin{array}{l}23 \cdot 0 \\
25 \cdot 0\end{array}$ & $29 \cdot 0$ & $\begin{array}{l}28 \cdot 0, \\
30 \cdot 0\end{array}$ & & $1 \cdot 4$ & $41 \cdot 2,1 \cdot 8$ & 0.9 & $0 \cdot 8,1 \cdot 0$ & 0.6 & $0 \cdot 4,0 \cdot 7$ & \\
\hline $\mathrm{BMI}\left(\mathrm{kg} / \mathrm{m}^{2}\right)$ & $21 \cdot 3$ & $3 \cdot 6$ & $21 \cdot 2$ & $3 \cdot 6$ & $21 \cdot 0$ & $3 \cdot 4$ & 0.566 & $21 \cdot 0$ & $3 \cdot 6$ & $21 \cdot 6$ & 3.9 & $20 \cdot 9$ & $3 \cdot 3$ & 0.131 & $21 \cdot 1$ & 3.7 & $21 \cdot 1$ & 3.7 & $21 \cdot 3$ & 3.4 & 0.773 \\
\hline Weight (kg) & $53 \cdot 3$ & $10 \cdot 4$ & $52 \cdot 8$ & $10 \cdot 2$ & 53.5 & $10 \cdot 0$ & 0.767 & $52 \cdot 4$ & $10 \cdot 3$ & $54 \cdot 1$ & $10 \cdot 8$ & $53 \cdot 2$ & $9 \cdot 4$ & 0.193 & $52 \cdot 2$ & $10 \cdot 4$ & $53 \cdot 6$ & $10 \cdot 7$ & $53 \cdot 7$ & $9 \cdot 5$ & 0.243 \\
\hline $\begin{array}{l}\text { Height }(\mathrm{cm}) \\
\text { Pagn }\end{array}$ & $158 \cdot 5$ & $5 \cdot 9$ & $158 \cdot 6$ & $6 \cdot 0$ & $159 \cdot 9$ & $5 \cdot 7$ & 0.007 & $157 \cdot 5$ & $6 \cdot 5$ & $158 \cdot 2$ & $6 \cdot 3$ & $159 \cdot 4$ & $5 \cdot 9$ & 0.004 & $157 \cdot 0$ & $6 \cdot 3$ & $159 \cdot 2$ & $6 \cdot 8$ & $158 \cdot 6$ & $5 \cdot 6$ & $<0.001$ \\
\hline $\begin{array}{l}\text { Parental educational level }(n, \%) \\
\leq 6 \text { years }\end{array}$ & & & & & & & & & & & & & & & & & & & & & \\
\hline$\leq 6$ years & 80 & 32.4 & 59 & $25 \cdot 4$ & 26 & 13.4 & & 87 & $32 \cdot 0$ & 49 & $24 \cdot 0$ & 29 & $14 \cdot 7$ & & 71 & $31 \cdot 4$ & 53 & $23 \cdot 7$ & 41 & $18 \cdot 4$ & \\
\hline $7-9$ years & 64 & $25 \cdot 9$ & 50 & $21 \cdot 6$ & 29 & $15 \cdot 0$ & & 64 & $23 \cdot 5$ & 39 & $19 \cdot 1$ & 40 & $20 \cdot 3$ & & 52 & $23 \cdot 0$ & 54 & $24 \cdot 1$ & 37 & $16 \cdot 6$ & \\
\hline $10-12$ years & 67 & $27 \cdot 1$ & 58 & $25 \cdot 0$ & 54 & $27 \cdot 8$ & & 70 & $27 \cdot 7$ & 59 & $28 \cdot 9$ & 50 & $25 \cdot 4$ & & 68 & $30 \cdot 1$ & 52 & $23 \cdot 2$ & 59 & & \\
\hline$>12$ years & 36 & $14 \cdot 6$ & 65 & $28 \cdot 0$ & 85 & $43 \cdot 8$ & $<0.001$ & 51 & $18 \cdot 8$ & 57 & $27 \cdot 9$ & 78 & $39 \cdot 6$ & $<0.001$ & 35 & $15 \cdot 5$ & 65 & $29 \cdot 0$ & 86 & $38 \cdot 6$ & $<0.001$ \\
\hline Physical activity $(n, \%)$ & & & & & & & & & & & & & & & & & & & & & \\
\hline Never & 73 & $29 \cdot 6$ & 64 & $27 \cdot 6$ & 34 & $17 \cdot 5$ & & 83 & 30.5 & 52 & $25 \cdot 5$ & 36 & $18 \cdot 3$ & & 64 & $28 \cdot 3$ & 57 & $25 \cdot 4$ & 50 & $22 \cdot 4$ & \\
\hline$\leq 1$ time/week & 74 & $30 \cdot 0$ & 66 & $28 \cdot 4$ & 45 & $23 \cdot 2$ & & 74 & $27 \cdot 2$ & 63 & $30 \cdot 9$ & 48 & $24 \cdot 4$ & & 61 & $27 \cdot 0$ & 68 & $30 \cdot 4$ & 56 & $25 \cdot 1$ & \\
\hline 2-3 times/week & 68 & $27 \cdot 5$ & 72 & $31 \cdot 0$ & 73 & $37 \cdot 6$ & & 77 & $28 \cdot 3$ & 59 & $28 \cdot 9$ & 77 & $39 \cdot 1$ & & 69 & $30 \cdot 5$ & 76 & $33 \cdot 9$ & 68 & $30 \cdot 5$ & \\
\hline$>3$ times/week & 27 & 10.9 & 27 & 11.6 & 39 & $20 \cdot 1$ & & 32 & $11 \cdot 8$ & 27 & $13 \cdot 2$ & 34 & $17 \cdot 3$ & & 27 & $12 \cdot 0$ & 21 & $9 \cdot 4$ & 45 & $20 \cdot 2$ & \\
\hline Missing information & 5 & $2 \cdot 0$ & 3 & 1.3 & 3 & 1.6 & 0.008 & 6 & $2 \cdot 2$ & 3 & 1.5 & 2 & $1 \cdot 0$ & 0.032 & 5 & $2 \cdot 2$ & 2 & 0.9 & 4 & 1.8 & 0.063 \\
\hline Total energy intake (kJ/d) & 9460 & 3376 & 10280 & 3121 & 11347 & 2611 & $<0.001$ & 10280 & 3255 & 10276 & 3481 & 10309 & 2694 & 0.994 & 10648 & 3540 & 10251 & 3155 & 9958 & 2741 & 0.059 \\
\hline rgy intake (kcal/d) & 2261 & 807 & 2457 & 746 & 2712 & 624 & $<0.001$ & 2457 & 778 & 2456 & 832 & 2464 & 644 & 0.994 & 2545 & 846 & 2450 & 754 & 2380 & 655 & 0.059 \\
\hline Ca inta & 956 & 431 & 1113 & 397 & 1316 & 399 & $<0.001$ & 990 & 436 & 1107 & 411 & 1291 & 396 & $<0.001$ & 1067 & 456 & 1084 & 431 & 1191 & 407 & 0.005 \\
\hline $\mathrm{Ca}$ intake (mg/4184 kJ (1000 kcal)) & 433 & 160 & 465 & 140 & 491 & 124 & $<0.001$ & 407 & 137 & 463 & 134 & 534 & 135 & $<0.001$ & 430 & 155 & 446 & 128 & 508 & 139 & $<0.001$ \\
\hline & & & & & & & & & & Boys & $s(13$ years & s old) & & & & & & & & & \\
\hline & & & MD pa & attern & & & & & & DASH & $\mathrm{H}$ diet & & & & & & OHS dieta & tary index & & & \\
\hline & $\begin{array}{r}1 \text { st te } \\
(n 2\end{array}$ & $\begin{array}{l}\text { ertile } \\
\text { 219) }\end{array}$ & $\begin{array}{r}2 n d \text { te } \\
(n 18\end{array}$ & $\begin{array}{l}\text { ertile } \\
86)\end{array}$ & $\begin{array}{r}3 r d \text { te } \\
(n 1\end{array}$ & $\begin{array}{l}\text { ertile } \\
86)\end{array}$ & & $\begin{array}{l}\text { 1st te } \\
(n 23\end{array}$ & $\begin{array}{l}\text { tertile } \\
\text { 238) }\end{array}$ & $\begin{array}{r}\text { 2nd te } \\
(n 1\end{array}$ & $\begin{array}{l}\text { tertile } \\
\text { 192) }\end{array}$ & $\begin{array}{r}\text { 3rd te } \\
(n 16\end{array}$ & $\begin{array}{l}\text { tertile } \\
161)\end{array}$ & & & $\begin{array}{l}\text { tertile } \\
196)\end{array}$ & $\begin{array}{r}2 \text { nd te } \\
(n 15\end{array}$ & $\begin{array}{l}\text { tertile } \\
\text { 199) }\end{array}$ & & $\begin{array}{l}\text { tertile } \\
196)\end{array}$ & \\
\hline & $\begin{array}{l}\text { Mean } \\
\text { or } n\end{array}$ & SD or $\%$ & $\begin{array}{l}\text { Mean } \\
\text { or } n\end{array}$ & SD or $\%$ & $\begin{array}{l}\text { Mean } \\
\text { or } n\end{array}$ & SD or $\%$ & $P$ & $\begin{array}{l}\text { Mean } \\
\text { or } n\end{array}$ & SD or $\%$ & $\begin{array}{l}\text { Mean } \\
\text { or } n\end{array}$ & SD or $\%$ & $\begin{array}{l}\text { Mean } \\
\text { or } n\end{array}$ & SD or $\%$ & $P$ & $\begin{array}{l}\text { Mean } \\
\text { or } n\end{array}$ & SD or $\%$ & $\begin{array}{l}\text { Mean } \\
\text { or } n\end{array}$ & SD or $\%$ & $\begin{array}{l}\text { Mean } \\
\text { or } n\end{array}$ & SD or $\%$ & $P$ \\
\hline Median score $\left(\mathrm{P}_{25}, \mathrm{P}_{75}\right)$ & 3.0 & $2 \cdot 0,4 \cdot 0$ & $6 \cdot 0$ & $5 \cdot 0,6 \cdot 0$ & $7 \cdot 0$ & $7 \cdot 0,8 \cdot 0$ & & $20 \cdot 0$ & $\begin{array}{l}18 \cdot 0 \\
21 \cdot 0\end{array}$ & $25 \cdot 0$ & $\begin{array}{l}23 \cdot 0 \\
26 \cdot 0\end{array}$ & $29 \cdot 0$ & $\begin{array}{l}27 \cdot 0 \\
30 \cdot 0\end{array}$ & & $1 \cdot 6$ & $61.4,1 \cdot 9$ & $1 \cdot 0$ & $0 \cdot 9,1 \cdot 1$ & 0.6 & $0.5,0.7$ & \\
\hline $\mathrm{BMI}\left(\mathrm{kg} / \mathrm{m}^{2}\right)$ & $21 \cdot 0$ & $3 \cdot 6$ & $20 \cdot 7$ & $3 \cdot 7$ & $20 \cdot 6$ & $3 \cdot 3$ & 0.619 & $20 \cdot 8$ & 3.5 & $20 \cdot 7$ & 3.7 & $20 \cdot 8$ & 3.3 & 0.945 & $21 \cdot 1$ & $3 \cdot 4$ & 20.5 & 3.6 & $20 \cdot 7$ & $3 \cdot 6$ & $0 \cdot 254$ \\
\hline Weight (kg) & $55 \cdot 5$ & $11 \cdot 8$ & $54 \cdot 7$ & $12 \cdot 8$ & $55 \cdot 1$ & $11 \cdot 3$ & 0.812 & $55 \cdot 2$ & $11 \cdot 8$ & $54 \cdot 6$ & $12 \cdot 5$ & $55 \cdot 6$ & 11.5 & 0.746 & $56 \cdot 4$ & $11 \cdot 6$ & $53 \cdot 5$ & $12 \cdot 1$ & $55 \cdot 5$ & $12 \cdot 0$ & 0.046 \\
\hline Height $(\mathrm{cm})$ & $162 \cdot 2$ & $7 \cdot 6$ & 161.9 & $8 \cdot 6$ & $163 \cdot 0$ & $7 \cdot 9$ & 0.385 & $162 \cdot 5$ & $7 \cdot 9$ & $161 \cdot 8$ & 8.0 & $162 \cdot 8$ & $8 \cdot 3$ & 0.452 & $163 \cdot 2$ & $7 \cdot 7$ & $161 \cdot 0$ & $8 \cdot 3$ & $163 \cdot 0$ & $7 \cdot 9$ & 0.010 \\
\hline Parents' years of schooling $(n, \%)$ & & & & & & & & & & & & & & & & & & & & & \\
\hline$\leq 6$ years & 53 & $24 \cdot 2$ & 40 & 21.5 & 17 & $9 \cdot 1$ & & 56 & $23 \cdot 5$ & 34 & $17 \cdot 7$ & 20 & 12.4 & & 50 & $25 \cdot 5$ & 41 & $20 \cdot 6$ & 19 & $9 \cdot 7$ & \\
\hline $7-9$ yea & 53 & 24 & 42 & $22 \cdot 6$ & 22 & $11 \cdot 8$ & & 61 & $25 \cdot 6$ & 37 & 19 & 19 & 11 & & 50 & $25 \cdot 5$ & 39 & $19 \cdot 6$ & 28 & $14 \cdot 3$ & \\
\hline $10-12$ years & 64 & $29 \cdot 2$ & 52 & $28 \cdot 0$ & 58 & $31 \cdot 2$ & & 65 & $27 \cdot 3$ & 57 & $29 \cdot 7$ & 52 & $32 \cdot 3$ & & 59 & $30 \cdot 1$ & 56 & $28 \cdot 1$ & 59 & $30 \cdot 1$ & \\
\hline$>12$ years & 49 & $22 \cdot 4$ & 52 & $28 \cdot 0$ & 89 & $47 \cdot 8$ & $<0.001$ & 56 & $23 \cdot 5$ & 64 & $33 \cdot 3$ & 70 & $43 \cdot 5$ & $<0.001$ & 37 & $18 \cdot 9$ & 63 & $31 \cdot 7$ & 90 & $45 \cdot 9$ & $<0.001$ \\
\hline Physical activity ( $n, \%)$ & & & & & & & & & & & & & & & & & & & & & \\
\hline Never & 22 & $10 \cdot 0$ & 19 & $10 \cdot 2$ & 16 & $8 \cdot 6$ & & 27 & $11 \cdot 3$ & 18 & $9 \cdot 4$ & 12 & $7 \cdot 4$ & & 18 & $9 \cdot 2$ & 25 & $12 \cdot 6$ & 14 & $7 \cdot 1$ & \\
\hline$\leq 1$ time/week & 42 & $19 \cdot 2$ & 26 & 14.0 & 28 & $15 \cdot 0$ & & 42 & 17.6 & 32 & $16 \cdot 7$ & 22 & $13 \cdot 7$ & & 32 & $16 \cdot 3$ & 32 & $16 \cdot 1$ & 32 & $16 \cdot 3$ & \\
\hline $2-3$ times $/ w$ & 60 & $27 \cdot 4$ & 66 & 35.5 & 65 & $35 \cdot 0$ & & 66 & $27 \cdot 7$ & 68 & $35 \cdot 4$ & 57 & $35 \cdot 4$ & & 55 & $28 \cdot 1$ & 64 & $32 \cdot 2$ & 72 & $36 \cdot 7$ & \\
\hline$>3 \mathrm{tim}$ & 84 & $38 \cdot 4$ & 73 & $39 \cdot 2$ & 69 & $37 \cdot 1$ & & 94 & $39 \cdot 5$ & 72 & 37.5 & 60 & $37 \cdot 3$ & & 83 & $42 \cdot 4$ & 77 & 38.7 & 66 & 33.7 & \\
\hline Missir & 11 & $5 \cdot 0$ & 2 & $1 \cdot 1$ & 8 & $4 \cdot 3$ & 0.280 & 9 & 3.8 & 2 & $1 \cdot 0$ & 10 & $6 \cdot 2$ & $0 \cdot 156$ & 8 & $4 \cdot 1$ & 1 & 0.5 & 12 & $6 \cdot 1$ & 0.038 \\
\hline intake $(\mathrm{kJ} / \mathrm{d})$ & 9761 & 3330 & 10452 & 264 & 11757 & 2615 & $<0.001$ & 10594 & 3167 & 10862 & 3163 & 10326 & 2653 & 0.259 & 10778 & 3222 & 10698 & 3059 & 10343 & 2807 & 0.314 \\
\hline Total energy intake (kcal/d) & 2333 & 796 & 2498 & 644 & 2810 & 625 & $<0.001$ & 2532 & 757 & 2596 & 756 & 2468 & 634 & 0.259 & 2576 & 770 & 2557 & 731 & 2472 & 671 & 0.314 \\
\hline Ca intake $(\mathrm{mg} / \mathrm{d})$ & 993 & 405 & 1119 & 360 & 1364 & 407 & $<0.001$ & 1009 & 382 & 1201 & 422 & 1295 & 414 & $<0.001$ & 1077 & 419 & 1152 & 413 & 1219 & 420 & 0.004 \\
\hline Ca intake (mg/4184 kJ (1000 kcal)) & 435 & 139 & 458 & 126 & 488 & 112 & $<0.001$ & 404 & 114 & 468 & 119 & 528 & 123 & $<0.001$ & 425 & 133 & 455 & 117 & 497 & 125 & $<0.001$ \\
\hline
\end{tabular}

$P_{25}$, 25th percentile; $P_{75}$, 75th percentile. 
Table 2 Bone mineral density (BMD) at 13 years old (baseline evaluation, 2003/2004) and at 17 years old (first follow-up evaluation, 2007/2008) according to tertile of adherence to the Mediterranean diet (MD) pattern, to the Dietary Approaches to Stop Hypertension (DASH) diet and to the Oslo Health Study (OHS) dietary index in Portuguese girls and boys, EPITeen cohort

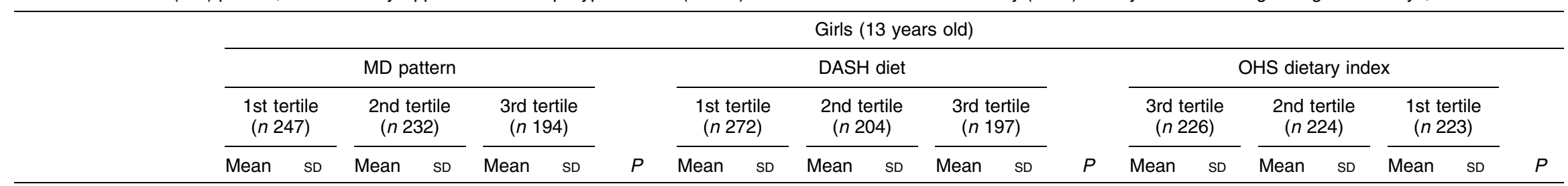

\begin{tabular}{llllllllllllllllllllllll}
\hline Baseline BMD $\left(\mathrm{g} / \mathrm{cm}^{2}\right)$ & 0.360 & 0.059 & 0.361 & 0.055 & 0.361 & 0.060 & 0.996 & 0.359 & 0.059 & 0.361 & 0.057 & 0.362 & 0.056 & 0.811 & 0.355 & 0.054 & 0.365 & 0.059 & 0.361 & 0.059 & 0.179
\end{tabular}

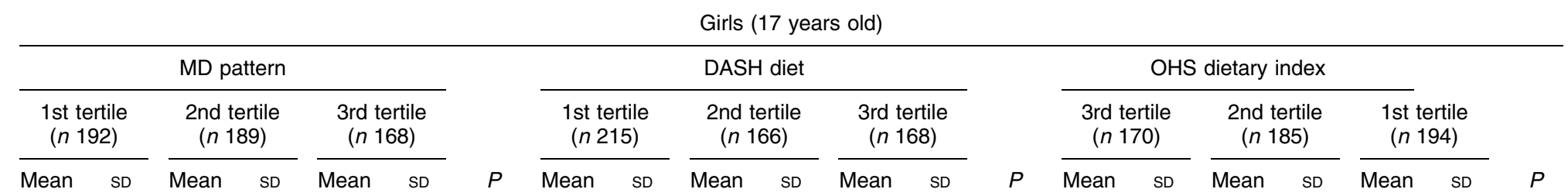

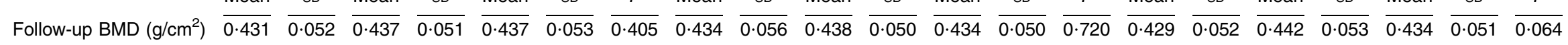

Boys (13 years old)

\begin{tabular}{|c|c|c|c|c|c|c|c|c|c|c|c|c|c|c|c|c|c|c|c|}
\hline \multicolumn{6}{|c|}{ MD pattern } & & \multicolumn{6}{|c|}{ DASH diet } & & \multicolumn{6}{|c|}{ OHS dietary index } \\
\hline $\begin{array}{r}1 \mathrm{st} t \\
(n-2\end{array}$ & & $\begin{array}{l}\text { 2nd t } \\
(n 1\end{array}$ & $\begin{array}{l}\text { rtile } \\
6 \text { ) }\end{array}$ & $\begin{array}{r}3 \mathrm{rd} \mathrm{t} \\
(n 1\end{array}$ & & \multirow[b]{2}{*}{$P$} & $\begin{array}{r}1 \mathrm{st} t \\
(n 2\end{array}$ & & $\begin{array}{l}\text { 2nd } t \\
(n 1\end{array}$ & & $\begin{array}{r}3 \mathrm{rd} \\
(n\end{array}$ & & \multirow[b]{2}{*}{$P$} & \multicolumn{4}{|c|}{$\begin{array}{c}\text { 2nd tertile } \\
(n \text { 199) }\end{array}$} & \multicolumn{2}{|c|}{$\begin{array}{c}\text { 1st tertile } \\
(n \text { 196) }\end{array}$} \\
\hline Mean & SD & Mean & SD & Mean & SD & & Mean & SD & Mean & SD & Mean & SD & & Mean & SD & Mean & SD & Mean & SD \\
\hline
\end{tabular}

$\begin{array}{llllllllllllllllllllllllllll}\text { Baseline BMD }\left(\mathrm{g} / \mathrm{cm}^{2}\right) & \overline{0.338} & \overline{0.047} & \overline{0.347} & \overline{0.052} & \overline{0.347} & \overline{0.053} & \overline{0.132} & \overline{0.342} & \overline{0.051} & \overline{0.344} & \overline{0.052} & \overline{0.346} & \overline{0.048} & \overline{0.720} & \overline{0.342} & \overline{0.051} & \overline{0.344} & \overline{0.050} & \overline{0.344} & \overline{0.050} & \overline{0.860}\end{array}$

\begin{tabular}{|c|c|c|c|c|c|c|c|c|c|c|c|c|c|c|c|c|c|c|c|c|}
\hline \multicolumn{21}{|c|}{ Boys (17 years old) } \\
\hline \multicolumn{6}{|c|}{ MD pattern } & \multirow[b]{3}{*}{$P$} & \multicolumn{6}{|c|}{ DASH diet } & \multicolumn{7}{|c|}{ OHS dietary index } & \multirow[b]{3}{*}{$P$} \\
\hline \multicolumn{2}{|c|}{$\begin{array}{c}\text { 1st tertile } \\
(n \text { 173) }\end{array}$} & \multicolumn{2}{|c|}{$\begin{array}{l}\text { 2nd tertile } \\
\quad(n \text { 147) }\end{array}$} & \multicolumn{2}{|c|}{$\begin{array}{c}\text { 3rd tertile } \\
(n 154)\end{array}$} & & \multicolumn{2}{|c|}{$\begin{array}{c}\text { 1st tertile } \\
(n \text { 187) }\end{array}$} & \multicolumn{2}{|c|}{$\begin{array}{l}\text { 2nd tertile } \\
\text { (n 154) }\end{array}$} & \multicolumn{2}{|c|}{$\begin{array}{c}\text { 3rd tertile } \\
(n \text { 133) }\end{array}$} & \multirow[b]{2}{*}{$P$} & \multicolumn{2}{|c|}{$\begin{array}{c}\text { 3rd tertile } \\
(n 151)\end{array}$} & \multicolumn{2}{|c|}{$\begin{array}{l}\text { 2nd tertile } \\
\quad(n \text { 168) }\end{array}$} & \multicolumn{2}{|c|}{$\begin{array}{l}\text { 1st tertile } \\
(n 155)\end{array}$} & \\
\hline Mean & SD & Mean & SD & Mean & SD & & Mean & SD & Mean & SD & Mean & SD & & Mean & SD & Mean & SD & Mean & SD & \\
\hline 0.450 & 0.074 & 0.448 & 0.075 & 0.458 & 0.076 & 0.510 & 0.455 & 0.074 & 0.452 & 0.079 & 0.448 & 0.072 & 0.679 & 0.456 & 0.076 & 0.451 & 0.076 & 0.450 & 0.074 & 0.748 \\
\hline
\end{tabular}

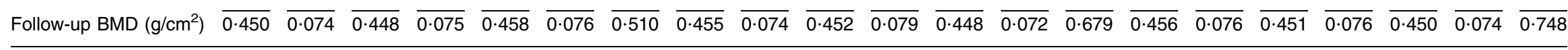


Table 3 Linear regression coefficients (95\% confidence intervals) for the cross-sectional and prospective associations between adherence to the Mediterranean diet (MD) pattern, to the Dietary Approaches to Stop Hypertension (DASH) diet and to the Oslo Health Study (OHS) dietary index and bone mineral density (BMD) in mg/cm ${ }^{2}$ among Portuguese girls and boys, EPITeen cohort

\begin{tabular}{|c|c|c|c|c|c|c|c|c|c|c|c|c|}
\hline & \multicolumn{12}{|c|}{ Outcome: $\mathrm{BMD}_{13}\left(\mathrm{mg} / \mathrm{cm}^{2}\right)$} \\
\hline & \multicolumn{4}{|c|}{ MD pattern } & \multicolumn{4}{|c|}{ DASH diet } & \multicolumn{4}{|c|}{ OHS dietary index } \\
\hline & $\begin{array}{c}\text { Crude } \\
\text { coefficient }\end{array}$ & $95 \% \mathrm{Cl}$ & $\begin{array}{l}\text { Adjusted } \\
\text { coefficient }^{*}\end{array}$ & $95 \% \mathrm{Cl}$ & $\begin{array}{c}\text { Crude } \\
\text { coefficient }\end{array}$ & $95 \% \mathrm{Cl}$ & $\begin{array}{l}\text { Adjusted } \\
\text { coefficient }^{\star}\end{array}$ & $95 \% \mathrm{Cl}$ & $\begin{array}{l}\text { Crude } \\
\text { coefficient }\end{array}$ & $95 \% \mathrm{Cl}$ & $\begin{array}{l}\text { Adjusted } \\
\text { coefficientt }\end{array}$ & $95 \% \mathrm{Cl}$ \\
\hline $\begin{array}{l}\text { Girls } \\
\text { Boys }\end{array}$ & $\begin{array}{r}-0.010 \\
0.192\end{array}$ & $\begin{array}{l}-0.215,0.194 \\
-0.008,0.392\end{array}$ & $\begin{array}{r}-0.019 \\
0.248\end{array}$ & $\begin{array}{r}-0.191,0.154 \\
0.052,0.444\end{array}$ & $\begin{array}{l}0.042 \\
0.070\end{array}$ & $\begin{array}{l}-0.055,0.140 \\
-0.020,0.161\end{array}$ & $\begin{array}{r}-0.005 \\
0.082\end{array}$ & $\begin{array}{l}-0.082,0.072 \\
-0.002,0.166\end{array}$ & $\begin{array}{l}-0.598 \\
-0.266\end{array}$ & $\begin{array}{l}-1 \cdot 406,0.210 \\
-0.963,0.431\end{array}$ & $\begin{array}{l}-0.293 \\
-0.597\end{array}$ & $\begin{array}{l}-0.932,0.347 \\
-1.278,0.084\end{array}$ \\
\hline
\end{tabular}

\begin{tabular}{|c|c|c|c|c|c|c|c|c|c|c|c|c|}
\hline & \multicolumn{4}{|c|}{ MD pattern } & \multicolumn{4}{|c|}{ DASH diet } & \multicolumn{4}{|c|}{ OHS dietary index } \\
\hline & $\begin{array}{c}\text { Crude } \\
\text { coefficient }\end{array}$ & $95 \% \mathrm{Cl}$ & $\begin{array}{l}\text { Adjusted } \\
\text { coefficient† }\end{array}$ & $95 \% \mathrm{Cl}$ & $\begin{array}{c}\text { Crude } \\
\text { coefficient }\end{array}$ & $95 \% \mathrm{Cl}$ & $\begin{array}{l}\text { Adjusted } \\
\text { coefficientt }\end{array}$ & $95 \% \mathrm{Cl}$ & $\begin{array}{c}\text { Crude } \\
\text { coefficient }\end{array}$ & $95 \% \mathrm{Cl}$ & $\begin{array}{l}\text { Adjusted } \\
\text { coefficientt }\end{array}$ & $95 \% \mathrm{Cl}$ \\
\hline $\begin{array}{l}\text { Girls } \\
\text { Boys }\end{array}$ & $\begin{array}{l}0.142 \\
0.061\end{array}$ & $\begin{array}{l}-0.062,0.346 \\
-0.265,0.388\end{array}$ & $\begin{array}{l}0 \cdot 046 \\
0 \cdot 158\end{array}$ & $\begin{array}{l}-0.158,0.250 \\
-0.185,0.501\end{array}$ & $\begin{array}{r}0.022 \\
-0.019\end{array}$ & $\begin{array}{l}-0 \cdot 077,0 \cdot 121 \\
-0 \cdot 170,0 \cdot 131\end{array}$ & $\begin{array}{l}-0.004 \\
-0.001\end{array}$ & $\begin{array}{l}-0.096,0.089 \\
-0.147,0.146\end{array}$ & $\begin{array}{r}-0.782 \\
0.821\end{array}$ & $\begin{array}{l}-1 \cdot 604,0 \cdot 040 \\
-0.380,2.022\end{array}$ & $\begin{array}{r}-0.538 \\
0.405\end{array}$ & $\begin{array}{l}-1 \cdot 311,0.235 \\
-0.806,1.617\end{array}$ \\
\hline
\end{tabular}

Outcome: annual BMD variation $\left(\mathrm{mg} / \mathrm{cm}^{2}\right.$ per year)

\begin{tabular}{|c|c|c|c|c|c|c|c|c|c|c|c|c|}
\hline & \multicolumn{4}{|c|}{ MD pattern } & \multicolumn{4}{|c|}{ DASH diet } & \multicolumn{4}{|c|}{ OHS dietary index } \\
\hline & $\begin{array}{l}\text { Crude } \\
\text { coefficient }\end{array}$ & $95 \% \mathrm{Cl}$ & $\begin{array}{l}\text { Adjusted } \\
\text { coefficientt }\end{array}$ & $95 \% \mathrm{Cl}$ & $\begin{array}{l}\text { Crude } \\
\text { coefficient }\end{array}$ & $95 \% \mathrm{Cl}$ & $\begin{array}{l}\text { Adjusted } \\
\text { coefficient }\end{array}$ & $95 \% \mathrm{Cl}$ & $\begin{array}{l}\text { Crude } \\
\text { coefficient }\end{array}$ & $95 \% \mathrm{Cl}$ & $\begin{array}{l}\text { Adjusted } \\
\text { coefficient† }\end{array}$ & $95 \% \mathrm{Cl}$ \\
\hline $\begin{array}{l}\text { Girls } \\
\text { Boys }\end{array}$ & $\begin{array}{r}0.028 \\
-0.019\end{array}$ & $\begin{array}{l}-0.025,0.081 \\
-0.091,0.053\end{array}$ & $\begin{array}{r}0.028 \\
-0.012\end{array}$ & $\begin{array}{l}-0.025,0.081 \\
-0.092,0.068\end{array}$ & $\begin{array}{r}0.002 \\
-0.026\end{array}$ & $\begin{array}{l}-0.023,0.027 \\
-0.059,0.007\end{array}$ & $\begin{array}{l}-0.002 \\
-0.026\end{array}$ & $\begin{array}{l}-0.026,0.022 \\
-0.060,0.008\end{array}$ & $\begin{array}{r}-0.049 \\
0.254\end{array}$ & $\begin{array}{l}-0.256,0.158 \\
-0.010,0.518\end{array}$ & $\begin{array}{r}-0.039 \\
0.204\end{array}$ & $\begin{array}{l}-0.240,0 \cdot 162 \\
-0.078,0 \cdot 487\end{array}$ \\
\hline
\end{tabular}

$\mathrm{BMD}_{13}$, bone mineral density $\left(\mathrm{mg} / \mathrm{cm}^{2}\right)$ at 13 years old; $\mathrm{BMD}_{17}$, bone mineral density $\left(\mathrm{mg} / \mathrm{cm}^{2}\right)$ at 17 years old.

Further adjustment of the linear regression coefficients for regular practice of physical activity and mean Ca intake did not change the results appreciably (data not shown).

in

tAdjusted for height and weight at 17 years of age, total energy intake, parental educational level and, in girls, age at menarche. 
regression coefficients and 95\% confidence intervals. Both crude and adjusted linear regressions coefficients confirm the lack of clear associations between the a priori dietary patterns studied and BMD at 13 years and longitudinal changes in BMD at 17 years in this sample of adolescents. Among males, higher adherence to the MD pattern was significantly associated with higher BMD at 13 years $(P=0 \cdot 013)$ but not to its annual variation. Further adjustment of the linear regression coefficients for adolescents' regular practice of physical activity and mean $\mathrm{Ca}$ intake did not change the results appreciably (data not shown).

\section{Discussion}

In the present study we observed no significant difference in annual variation of BMD, between 13 and 17 years of age, by adherence to the different a priori dietary patterns. However, among boys, a significant linear trend towards increased BMD at 13 years with increasing adherence to the MD pattern was observed.

Little research has been done examining the relationship between a priori-defined dietary patterns and bone quality $^{(20,31-34)}$. To our knowledge, the present study is the first one to explore both cross-sectional and prospective associations between a priori-defined dietary patterns and BMD during the adolescent growth spurt.

We identified two studies examining the association between adherence to the MD pattern and $\mathrm{BMD}^{(31,33)}$. Adherence to the MD was not significantly related to total body bone mineral content or to BMD at the lumbar spine in adult women ${ }^{(31)}$. Also no evidence of an association between BMD or bone mineral content and adherence to this pattern was found in a sample of young adults ${ }^{(33)}$. The association between adherence to the DASH diet and bone metabolism was studied as a 3-month DASH intervention study in adults and this pattern significantly reduced bone turnover ${ }^{(18)}$.

The MD and DASH diets are characterized by high intakes of fruit and vegetables, wholegrain bread and cereals, pulses, nuts, dairy products and fish ${ }^{(29)}$. Although these patterns value the consumption of alkali-forming food groups that have been associated with higher BMD (i.e. fruit and vegetables) ${ }^{(35-37)}$, they also include increased quantities of acid-forming foods (i.e. cereals, pulses, nuts and dairy products). The latter can change the acid-base balance and may preclude expression of the beneficial effects of fruit and vegetables ${ }^{(38,39)}$.

One of the main limitations of the a priori dietary patterns approach is related to the use of dietary guidelines that generally are not disease specific and the adherence to them may reduce the risk of some diseases but not others ${ }^{(40)}$. Therefore, we additionally studied the association between BMD and the OHS dietary index. Although this pattern was associated with BMD in a sample of adults ${ }^{(20)}$, our study found no indication of such an association among adolescents.

In our study, the ascertainment of usual diet was done using an FFQ and, as with any method to assess dietary intake, under-reporting of usual intake is possible because of social desirability bias ${ }^{(41,42)}$, particularly in overweight and obese individuals ${ }^{(43,44)}$. Differential under-reporting of diet components is a relevant limitation to pattern analysis ${ }^{(41)}$. For example, diet under-reporting of foods high in fat or sugar, such as fast foods, pastries or sweetened beverages, will be reflected in higher scores in the MD and DASH indices and in lower scores in the OHS dietary index. Additionally, there could be inherent imprecision associated with the option of not quantifying food portion sizes. However, research has shown that the majority of variation in food intakes is captured by frequency of consumption ${ }^{(45)}$. The lack of validation of the FFQ in this adolescent population may also be a limitation. However, the FFQ had been previously validated in the adult population of the same city ${ }^{(46)}$.

Moreover, in our study dietary intake was evaluated at baseline and we do not know whether adherence to dietary patterns changed throughout the follow-up. Although some studies have been providing evidence that variations of dietary patterns occur during adolescence ${ }^{(47,48)}$, it is also likely that dietary patterns remain relatively constant over time ${ }^{(49-52)}$.

Taking into account the weak and few significant associations found in the present study, we hypothesize that dietary patterns may not be the primary factor in determining BMD in this age group. It is possible that dietary patterns may not have the impact on BMD in adolescents as they do in adult populations. One possible explanation may be the fact that, contrary to what happens in heterogeneous samples of adults, our sample of adolescents is very homogeneous, since they were born in the same year and share the urban environment of Porto city, making it much more difficult to find consistent associations between diet and BMD.

In our study we observed gender differences in the association between adherence to the MD pattern and BMD, possibly attributable to sex-specific difference in BMD response to biological and environmental determinants at this age ${ }^{(53)}$. Indeed, it is possible that the environmental determinants of bone development during adolescence differ according to sex as was previously described for the relationship between physical activity and $\mathrm{BMD}^{(54)}$.

In order to optimize feasibility and to minimize radiation exposure we used forearm BMD measured by DXA. The DXA-derived BMD is based on the two-dimensional projected area of a three-dimensional structure which does not capture true volumetric density or bone geometry ${ }^{(55)}$. However, BMD remains a valid index of bone quality and DXA-derived measures have been shown to predict fracture risk accurately, which is ultimately the 
goal of bone quality assessment ${ }^{(56)}$. Since we studied bone quality only by examining forearm BMD measured with DXA, we cannot rule out the possibility that other measures of bone quality are associated with the dietary patterns considered.

One possible confounder of the association between dietary patterns and BMD is body size. Although we did not find substantial variation in mean BMI, weight and height between different classes of adherence to the dietary patterns studied, which may be related to the aforementioned limitations of the dietary assessment method, there is a well-documented important weightdependent positive association between adiposity and bone strength ${ }^{(57)}$ that has been found in a previous work evaluating the girls of this cohort ${ }^{(58)}$. For this reason we found it essential to account for confounding by body size in the present analysis.

Owing to the difficulty in ensuring privacy at school, adolescents' pubertal development by the Tanner criteria ${ }^{(28)}$ was not assessed. This may be a limitation since it is possible that the higher height observed in boys of the third tertile of the MD pattern may reflect greater maturity. However, for a sub-sample of 121 boys, we identified no significant difference in mean level of serum collagen type 1 cross-linked C-telopeptide, a marker of bone resorption that has been associated with skeletal maturation ${ }^{(59)}$, according to tertile of MD pattern adherence. In girls, age at menarche was recorded as an indicator of pubertal development in girls.

Physical activity is also a possible confounder of the association studied, but in the present study it was not consistently associated with either dietary patterns or BMD (data not shown). One possible explanation is the low validity of self-reported information to assess physical activity. However, the most probable reason is the previously described high levels of sedentary behaviour in this population ${ }^{(22)}$, which probably situated the vast majority of the sample below the threshold level for exercise-induced bone formation. In fact, there is evidence that light or moderate physical activity is not associated with bone properties ${ }^{(60)}$. Although we cannot exclude that physical activity might modify the effect between diet and BMD, the further adjustment to this confounder did not change the results.

As with any observational analysis, it is possible that other confounders that were not measured in the study or error in the measurement of the existing confounders could lead to residual confounding that could not be accounted for. However, we controlled for other potential confounders, which did not substantially affect our estimates.

Another limitation is the large number of comparisons made in the present study, which makes it difficult to rule out the role of chance in any one result. However, adjustment for multiple comparisons by the Bonferroni method did not substantially change the results ${ }^{(61)}$.
Although the dietary patterns approach has certain advantages over traditional methods of examining the relationship between diet and health outcomes, results can be more challenging to interpret. In fact, more than the food combinations consumed, dietary patterns probably reflect individual food preferences modulated by a mix of genetic, cultural, social, health, environmental, lifestyle and economic determinants ${ }^{(12)}$. As a result of this complexity, mechanisms to explain the observed associations are not clarified by the present results. In fact, more healthy dietary patterns are often reported with a constellation of other desirable health behaviours, thus confounding the pattern and health association ${ }^{(12)}$.

\section{Conclusions}

We did not find consistent or strong associations between dietary patterns defined a priori and forearm BMD in early or late adolescence. The selected dietary patterns may not capture the elements of diet that are truly important in determining adolescent bone quality or, given the overall adequacy of nutrient intake in highincome populations, dietary patterns may not add substantially to other determinants of BMD at this age.

\section{Acknowledgements}

Sources of funding: This work was supported by the Portuguese Foundation for Science and Technology (PTDC/SAU-ESA/108407/2008, PTDC/SAU-EPI/115254/ 2009 and FCOMP-01-FEDER-0124-015750). Conflicts of interest: The authors have no conflicts of interest to disclose. Authors' contributions: T.M. was responsible for data analysis and interpretation and for drafting the first version of the paper. R.L. contributed substantially to the interpretation of results and critically revised the manuscript. E.R. supervised the design of the study as well as the data collection protocols, contributed to the interpretation of data and critically revised the manuscript for intellectual content. H.B. supervised the conception and the design of the study as well as the interpretation of results and critically revised the paper for intellectual content. Acknowledgements: The authors gratefully acknowledge Associação Portuguesa de Osteoporose (APO) for making the bone densitometry equipment available for both evaluations.

\section{References}

1. World Health Organization (2003) The Burden of Musculoskeletal Conditions at the Start of the New Millenium. Report of a WHO Scientific Group. WHO Technical Report Series no. 919. Geneva: WHO; available at http://whqlibdoc.who. int/trs/WHO_TRS_919.pdf 
2. Lidgren L, Smolen J, Bentley G et al. (2005) European Action Towards Better Musculoskeletal Health: A Public Health Strategy to Reduce the Burden of Musculoskeletal Conditions. Lund: The Bone \& Joint Decade, Department of Orthopedics, University Hospital; available at http:// www.boneandjointdecade.org/Default.aspx? contId $=534$

3. Heaney RP, Abrams S, Dawson-Hughes B et al. (2000) Peak bone mass. Osteoporos Int 11, 985-1009.

4. Javaid MK \& Cooper C (2002) Prenatal and childhood influences on osteoporosis. Best Pract Res Clin Endocrinol Metab 16, 349-367.

5. Bachrach LK (2001) Acquisition of optimal bone mass in childhood and adolescence. Trends Endocrinol Metab 12, 22-28.

6. Ilich JZ \& Kerstetter JE (2000) Nutrition in bone health revisited: a story beyond calcium. J Am Coll Nutr 19 , 715-737.

7. Cashman KD (2007) Diet, nutrition, and bone health. JNutr 137, 11 Suppl., 2507S-2512S.

8. Rizzoli R (2008) Nutrition: its role in bone health. Best Pract Res Clin Endocrinol Metab 22, 813-829.

9. Weaver CM (2008) The role of nutrition on optimizing peak bone mass. Asia Pac J Clin Nutr 17, Suppl. 1, 135-137.

10. Rizzoli R, Bonjour JP \& Chevalley T (2010) Dietary protein and bone mass accrual. In: Nutritional Influences on Bone Health, pp. 1-8 [P Burckhardt, B Dawson-Hughes and C Weaver, editors]. London: Springer-Verlag.

11. Kontogianni MD \& Yiannakouris N (2009) Diet and bone health - the perspective of dietary pattern analysis. Eur Musculoskelet Rev 4, 73-74.

12. Kant AK (2004) Dietary patterns and health outcomes. J Am Diet Assoc 104, 615-635.

13. Bach A, Serra-Majem L, Carrasco JL et al. (2006) The use of indexes evaluating the adherence to the Mediterranean diet in epidemiological studies: a review. Public Health Nutr 9 , 132-146.

14. Prentice A, Schoenmakers I, Laskey MA et al. (2006) Nutrition and bone growth and development. Proc Nutr Soc 65, 348-360.

15. Sacks FM, Svetkey LP, Vollmer WM et al. (2001) Effects on blood pressure of reduced dietary sodium and the Dietary Approaches to Stop Hypertension (DASH) diet. DASHSodium Collaborative Research Group. N Engl J Med 344, 3-10.

16. Karanja NM, Obarzanek E, Lin PH et al. (1999) Descriptive characteristics of the dietary patterns used in the Dietary Approaches to Stop Hypertension Trial. DASH Collaborative Research Group. J Am Diet Assoc 99, 8 Suppl., S19-S27.

17. Appel LJ, Moore TJ, Obarzanek E et al. (1997) A clinical trial of the effects of dietary patterns on blood pressure. DASH Collaborative Research Group. $N$ Engl J Med 336, $1117-1124$.

18. Lin PH, Ginty F, Appel LJ et al. (2003) The DASH diet and sodium reduction improve markers of bone turnover and calcium metabolism in adults. J Nutr 133, 3130-3136.

19. Hostmark AT (2010) The Oslo Health Study: a dietary index estimating high intake of soft drinks and low intake of fruits and vegetables was positively associated with components of the metabolic syndrome. Appl Physiol Nutr Metab 35, 816-825.

20. Hostmark AT, Sogaard AJ, Alvaer K et al. (2011) The oslo health study: a dietary index estimating frequent intake of soft drinks and rare intake of fruit and vegetables is negatively associated with bone mineral density. J Osteoporos 2011, 102686.

21. Shepherd R \& Dennison CM (1996) Influences on adolescent food choice. Proc Nutr Soc 55, 345-357.

22. Ramos E \& Barros H (2007) Family and school determinants of overweight in 13-year-old Portuguese adolescents. Acta Paediatr 96, 281-286.
23. Willett WC, Sampson L, Stampfer MJ et al. (1985) Reproducibility and validity of a semiquantitative food frequency questionnaire. Am J Epidemiol 122, 51-65.

24. Lopes C, Aro A, Azevedo A et al. (2007) Intake and adipose tissue composition of fatty acids and risk of myocardial infarction in a male Portuguese community sample. $J \mathrm{Am}$ Diet Assoc 107, 276-286.

25. Ramos E (2006) Health determinants in Porto adolescents. PhD Thesis, University of Porto.

26. Silva D, Rego C, Guerra A et al. (2004) Characterization of food habits and comparative study between two methods of food assessment in adolescents. Rev Aliment Hum 10, $33-40$.

27. Araujo J, Severo M, Lopes C et al. (2011) Food sources of nutrients among 13-year-old Portuguese adolescents. Public Health Nutr 14, 1970-1978.

28. Tanner JM (1962) Growth at Adolescence, 2nd ed. Oxford: Blackwell Scientific Publications.

29. Serra-Majem L, Ribas L, Ngo J et al. (2004) Food, youth and the Mediterranean diet in Spain. Development of KIDMED, Mediterranean Diet Quality Index in children and adolescents. Public Health Nutr 7, 931-935.

30. Fung TT, Chiuve SE, McCullough ML et al. (2008) Adherence to a DASH-style diet and risk of coronary heart disease and stroke in women. Arch Intern Med 168, 713-720.

31. Kontogianni MD, Melistas L, Yannakoulia M et al. (2009) Association between dietary patterns and indices of bone mass in a sample of Mediterranean women. Nutrition 25, 165-171.

32. Hamidi M, Tarasuk V, Corey P et al. (2011) Association between the Healthy Eating Index and bone turnover markers in US postmenopausal women aged $\geq 45$ y. $A m J$ Clin Nutr 94, 199-208.

33. Whittle CR, Woodside JV, Cardwell CR et al. (2012) Dietary patterns and bone mineral status in young adults: the Northern Ireland Young Hearts Project. Br J Nutr 108, 1494-1504.

34. Zagarins SE, Ronnenberg AG, Gehlbach SH et al. (2012) Are existing measures of overall diet quality associated with peak bone mass in young premenopausal women? J Hum Nutr Diet 25, 172-179.

35. Prynne CJ, Mishra GD, O'Connell MA et al. (2006) Fruit and vegetable intakes and bone mineral status: a cross sectional study in 5 age and sex cohorts. Am J Clin Nutr 83, 1420-1428.

36. Vatanparast H, Baxter-Jones A, Faulkner RA et al. (2005) Positive effects of vegetable and fruit consumption and calcium intake on bone mineral accrual in boys during growth from childhood to adolescence: the University of Saskatchewan Pediatric Bone Mineral Accrual Study. Am J Clin Nutr 82, 700-706.

37. McGartland CP, Robson PJ, Murray LJ et al. (2004) Fruit and vegetable consumption and bone mineral density: the Northern Ireland Young Hearts Project. Am J Clin Nutr 80, 1019-1023.

38. Remer T \& Manz F (1995) Potential renal acid load of foods and its influence on urine pH. J Am Diet Assoc 95, 791-797.

39. Bushinsky DA (2001) Acid-base imbalance and the skeleton. Eur J Nutr 40, 238-244.

40. Michels KB \& Schulze MB (2005) Can dietary patterns help us detect diet-disease associations? Nutr Res Rev 18, 241-248.

41. Togo P, Osler M, Sorensen TI et al. (2001) Food intake patterns and body mass index in observational studies. Int $J$ Obes Relat Metab Disord 25, 1741-1751.

42. Livingstone MB \& Black AE (2003) Markers of the validity of reported energy intake. J Nutr 133, Suppl. 3, 895S-920S.

43. Heitmann BL \& Lissner L (1995) Dietary underreporting by obese individuals - is it specific or non-specific? BMJ $\mathbf{3 1 1}$, 986-989. 
44. Newby PK (2007) Are dietary intakes and eating behaviors related to childhood obesity? A comprehensive review of the evidence. J Law Med Ethics 35, 35-60.

45. Noethlings U, Hoffmann K, Bergmann MM et al. (2003) Portion size adds limited information on variance in food intake of participants in the EPIC-Potsdam study. J Nutr 133, 510-515.

46. Lopes C (2000) Dietary factors and myocardial infarction: a community-based case-control study. PhD Thesis, University of Porto.

47. Madruga SW, Araujo CL, Bertoldi AD et al. (2012) Tracking of dietary patterns from childhood to adolescence. Rev Saude Publica 46, 376-386.

48. Patterson E, Warnberg J, Kearney J et al. (2009) The tracking of dietary intakes of children and adolescents in Sweden over six years: the European Youth Heart Study. Int J Behav Nutr Phys Act 6, 91.

49. Cutler GJ, Flood A, Hannan P et al. (2009) Major patterns of dietary intake in adolescents and their stability over time. J Nutr 139, 323-328.

50. te Velde SJ, Twisk JW \& Brug J (2007) Tracking of fruit and vegetable consumption from adolescence into adulthood and its longitudinal association with overweight. Br J Nutr 98, 431-438.

51. Mikkila V, Rasanen L, Raitakari OT et al. (2005) Consistent dietary patterns identified from childhood to adulthood: the cardiovascular risk in Young Finns Study. Br J Nutr $\mathbf{9 3}$ 923-931.

52. Oellingrath IM, Svendsen MV \& Brantsaeter AL (2011) Tracking of eating patterns and overweight - a follow-up study of Norwegian schoolchildren from middle childhood to early adolescence. Nutr J 10, 106.
53. Macdonald H, Kontulainen S, Petit M et al. (2006) Bone strength and its determinants in pre- and early pubertal boys and girls. Bone 39, 598-608.

54. Weeks BK \& Beck BR (2010) The relationship between physical activity and bone during adolescence differs according to sex and biological maturity. J Osteoporos 2010, 546593.

55. Crabtree N \& Ward K (2009) Bone densitometry: current status and future perspectives. Endocr Dev 16, 58-72.

56. Flynn J, Foley S \& Jones G (2007) Can BMD assessed by DXA at age 8 predict fracture risk in boys and girls during puberty?: an eight-year prospective study. J Bone Miner Res 22, 1463-1467.

57. Zhao LJ, Jiang H, Papasian CJ et al. (2008) Correlation of obesity and osteoporosis: effect of fat mass on the determination of osteoporosis. J Bone Miner Res 23, $17-29$.

58. Lucas R, Ramos E, Severo M et al. (2011) Potential for a direct weight-independent association between adiposity and forearm bone mineral density during adolescence. Am J Epidemiol 174, 691-700.

59. Silva CC, Goldberg TB, Nga HS et al. (2011) Impact of skeletal maturation on bone metabolism biomarkers and bone mineral density in healthy Brazilian male adolescents. $J$ Pediatr (Rio J) 87, 450-456.

60. Sayers A, Mattocks C, Deere K et al. (2011) Habitual levels of vigorous, but not moderate or light, physical activity is positively related to cortical bone mass in adolescents. J Clin Endocrinol Metab 96, E793-E802.

61. Rothman KJ (1990) No adjustments are needed for multiple comparisons. Epidemiology 1, 43-46. 